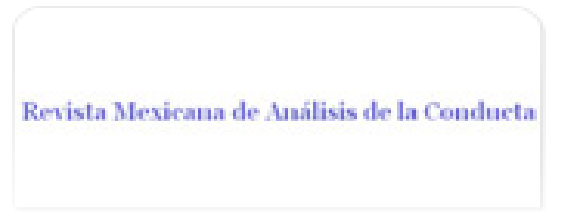

Revista Mexicana de Análisis de la Conducta ISSN: 0185-4534

editora@rmac-mx.org

Sociedad Mexicana de Análisis de la Conducta México

Flores Aguirre, Carlos Javier; Mateos Morfín, Laura Rebeca

Tiempo relativo, elección demorada y demora de reforzamiento en discriminación condicional Revista Mexicana de Análisis de la Conducta, vol. 35, núm. 1, junio, 2009, pp. 135-149

Sociedad Mexicana de Análisis de la Conducta

Guadalajara, México

Disponible en: http://www.redalyc.org/articulo.oa?id=59311416008

- Cómo citar el artículo

- Número completo

- Más información del artículo

- Página de la revista en redalyc.org

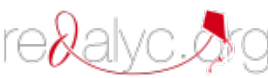

Sistema de Información Científica

Red de Revistas Científicas de América Latina, el Caribe, España y Portugal Proyecto académico sin fines de lucro, desarrollado bajo la iniciativa de acceso abierto 


\title{
TIEMPO RELATIVO, ELECCIÓN DEMORADA Y DEMORA DE REFORZAMIENTO EN DISCRIMINACIÓN CONDICIONAL
}

\author{
RELATIVE TIME, CHOICE DELAY AND DELAY OF REINFORCEMENT \\ IN CONDITIONAL DISCRIMINATION \\ CARLOS JAVIER FLORES AGUIRRE \\ Y LAURA REBECA MATEOS MORFÍN ${ }^{1}$ \\ CENTRO DE ESTUDIOS E INVESTIGACIONES EN COMPORTAMIENTO \\ UNIVERSIDAD DE GUADALAJARA
}

\begin{abstract}
RESUMEN
Algunos trabajos han reportado resultados inconsistentes sobre los efectos de alargar la demora de reforzamiento y el intervalo de retención bajo diferentes procedimientos en los que se varía el intervalo entre reforzadores (e.g., Schaal, Schuh \& Branch, 1992; Shahan \& Lattal, 2005; Williams, 1998, 2003). El estudio se diseñó con el propósito de evaluar los efectos conjuntos de variar la demora de reforzamiento y el intervalo de retención en situaciones en las que se mantenía o variaba en intervalo entre ensayos (IEE). Se entrenó a ratas en una tarea de discriminación condicional. En el Experimento 1 se incrementó el IEE para un grupo (IEE variable), mientras que para otro grupo la duración del IEE fue constante, concurrentemente se incrementó el intervalo entre el estímulo y la oportunidad para responder (intervalo de retención). Los efectos de alargar la duración del intervalo de retención fueron menores en el grupo con un IEE variable. El Experimento 2 fue una réplica del Exp.
\end{abstract}

1. Los autores dedican este trabajo al Dr. Emilio Ribes por sus 65 años celebrados el 24 de junio del presente. Una versión preliminar de este trabajo se presentó en el XXIX Internacional Congress of Psychology. Berlin, Alemania, Julio de 2008. La correspondencia puede enviarse a cualquiera de los autores a: Centro de Estudios e Investigaciones en Comportamiento, Francisco de Quevedo No. 180, Col. Arcos Vallarta, Guadalajara, Jalisco, México, 44130., e-mail carlos.flores@cucba. udg.mx. rebecamateos@gmail.com

Recibido: Enero 23, 2009. Revisado: Febrero 18, 2009. Aceptado: Mayo 11, 2009 
1 excepto que se incrementó la demora de reforzamiento $(0,2,4$ y 8 s). Se encontró que el porcentaje de respuestas correctas se mantuvo constante ante los diferentes valores de demora de reforzamiento. La ocurrencia del efecto de tiempo relativo en el Experimento 1 y su ausencia en el Experimento 2 sugieren que los efectos de los diferentes tipos de demora (intervalo de retención y demora de reforzamiento) dependen de diferentes procesos psicológicos, los resultados se discuten atendiendo a las diferencias entre los procedimientos empleados y en la generalidad del efecto de tiempo relativo.

Palabras clave: Tiempo relativo, demora de reforzamiento, discriminación condicional, precisión, ratas.

\begin{abstract}
Some recent studies reported inconsistent results about the effects of delay of reinforcement and retention interval in different procedures varying the intereinforcer interval (e.g., Schaal, Schuh \& Branch, 1992; Shahan \& Lattal, 2005; Williams, 1998, 2003). The study was designed to evaluate the effects of delay of reinforcement and the retention interval in different conditions, with an intertrial interval constant or variable. Rats were trained on a conditional discrimination procedure. In Experiment 1 the intertrial interval (ITI) was increased for a group (ITI variable), while for another group the ITI duration was constant, concurrently the stimulus-choice interval (retention interval) was increased. The effects of retention interval were smaller with longer ITI's (variable group). Experiment 2 was a reply of Exp. 1, except the delay of reinforcement was increased $(0,2,4$ and $8 \mathrm{~s})$. The percentage of correct responses was constant in all delay values. The occurrence of relative time effects in Experiment 1, but not in Experiment 2, suggest that different types of delay intervals (retention interval and delay of reinforcement) depend upon different psychological processes. The results are discussed in terms of differences in procedures and the generality of relative time effect.
\end{abstract}

Keywords: Relative time, delay of reinforcement, conditional discrimination, accuracy, rats.

Williams (1998) planteó que los efectos de algunas variables temporales (e.g., demora de reforzamiento, intervalo entre estímulos, etc.) pueden ser regulados por los valores o duraciones de otras variables (e.g., intervalo entre ensayos, intervalo entre reforzadores), efecto al que se le conoce en la literatura como "Efecto de Tiempo Relativo" (ETR), la generalidad de este efecto se ha reportado teniendo como variables dependientes a la tasa de respuesta, el porcentaje 
de respuestas correctas, etc. (e.g., Gibbon, Baldock, Locurto, Gold \& Terrace, 1977; Gibbon \& Balsam, 1981; Roberts \& Kraemer, 1982).

Específicamente, se puede decir que el ETR se identifica cuando los efectos "disruptores" de una variable temporal (e.g., intervalo entre estímulos o demora de reforzamiento) se ven aminorados cuando el contexto temporal entre reforzadores es de mayor duración (i.e. mayor espaciamiento entre los ensayos, mayor duración del intervalo entre reforzadores).

Este efecto se ha documentado utilizando tareas que implican contingencias estímulo-estímulo (e.g., Gibbon, Baldock, Locurto, Gold \& Terrace, 1977; Gibbon \& Balsam, 1981), como en procedimientos que implican tanto contingencias estímulo-estímulo como contingencias respuesta-estímulo (e.g., Maki, Moe \& Bierly, 1977; Roberts \& Kraemer, 1982).

Utilizando una tarea de discriminación condicional, Williams (1998, Exp. 1) reportó el ETR cuando varió la duración del intervalo de demora entre la señal condicional y la oportunidad para responder. Sin embargo, no encontró el mismo efecto cuando varió la demora de reforzamiento (Exp. 2). En el Experimento 1 encontró que las ratas entrenadas con un IEE de $45 \mathrm{~s}$ tuvieron una tasa de adquisición más rápida con demoras de 2 y $4 \mathrm{~s}$ que ratas entrenadas con un IEE de menor duración (15 s). En el Experimento 2 replicó el mismo procedimiento que en el experimento anterior pero variando la demora de reforzamiento. Reportó que las tasas de adquisición con demoras de reforzamiento de 2 y 6 s no se vieron modificadas por la duración del IEE.

Esta diferencia en los resultados obtenidos por Williams (1998) llevó a Shahan y Lattal (2005) a identificar el ETR pero variando la demora de reforzamiento no señalada en programas múltiples de intervalo variable (IV) con diferentes duraciones del intervalo entre reforzadores en cada componente (Exp. 1 Mult IV 15-s IV 90-s IV540-s y Exp. 2 Mult IV 20-s IV 120-s).

Shahan y Lattal (2005) reportaron que la demora de reforzamiento resultó en decrementos en las tasas de respuesta en los diferentes componentes del programa múltiple, no observando diferencias en el decremento entre las tasas de respuesta en los componentes con mayor o menor frecuencia de reforzamiento.

Schaal, Schuh y Branch (1992) reportaron el ETR variando la demora de reforzamiento señalada en programas de intervalo variable; mientras que Shahan y Lattal (2005) utilizando el mismo programa pero con demoras no señaladas no encontraron el mismo efecto. La discrepancia en los resultados reportados puede obedecer a las diferencias entre las tareas, sujetos, procedimientos y variables dependientes empleadas en los diferentes estudios.

Los estudios de Schaal, Schuh y Branch (1992) y Shahan y Lattal (2005) se realizaron utilizando programas múltiples de reforzamiento y variando la demora de reforzamiento (señalada y no señalada, respectivamente); mientras que el estudio de Williams (1998) se realizó utilizando un procedimiento 
de discriminación condicional y variando tanto la demora de reforzamiento como la demora entre la señal condicional y la oportunidad para la respuesta (intervalo de retención). En los estudios de Schaal, et. al. y de Shahan y Lattal, la variable dependiente fue la tasa de respuesta, mientras que en el estudio reportado por Williams (1998) la variable dependiente fue la precisión del responder (i.e. porcentaje de respuesta correctas). Adicionalmente, en algunos trabajos se ha reportado el ETR bajo condiciones de adquisición (Balsam, 1984; Bueno \& Álvarez, 2001; Yin, Barnet \& Miller, 1994), mientras que en otros ha sido bajo condiciones de mantenimiento.

Es posible que la ausencia del ETR bajo condiciones degradadas de reforzamiento (demora de reforzamiento) se deba a su evaluación sobre condiciones de adquisición (Williams, 1998, Exp. 2 y Exp. 3), es por ello que resulta necesario evaluar los efectos de la demora de reforzamiento bajo condiciones de mantenimiento empleando la misma tarea reportada por Williams. Aunque existen reportes del ETR bajo condiciones de mantenimiento éste se ha reportado utilizando procedimientos de igualación de la muestra y palomas como sujetos (e.g., Roberts \& Kraemer, 1982). La posible identificación de los efectos de tiempo relativo en condiciones diferentes a las de adquisición, permitirían extender su generalidad a condiciones de mantenimiento y su generalidad entre especies, procedimientos y variables dependientes. El presente estudio se diseñó con el propósito de evaluar bajo condiciones de mantenimiento, los efectos conjuntos de variar la duración del intervalo entre ensayos y el intervalo de demora entre la señal condicional y la oportunidad para responder (Experimento 1), así como la variación conjunta de la demora de reforzamiento y el intervalo entre ensayos (Experimento2), sobre el porcentaje de respuestas correctas en ratas en una tarea de discriminación condicional.

\section{EXPERIMENTO 1 \\ MÉTODO}

\section{Sujetos}

Se utilizaron 8 ratas Wistar de tres meses de edad al inicio del estudio e ingenuas experimentalmente. Los animales se mantuvieron en un régimen de privación de agua por un periodo promedio de $23.5 \mathrm{~h}$ diarias y con acceso libre al alimento en su caja habitación.

\section{Aparatos}

Se utilizaron 4 cámaras experimentales para ratas marca MED (ENV-008), cada caja estuvo equipada con un dispensador de agua (ENV-202M), dos 
palancas de respuesta y una tecla translúcida de $2.5 \mathrm{~cm}$ de diámetro que se iluminó de color blanco. Las palancas se encontraban a $2.5 \mathrm{~cm}$ del piso y requirieron de una fuerza de $25 \mathrm{gr}$ para cerrar el microswitch. Como reforzador se utilizó una gota de agua de $0.01 \mathrm{cc}$ que se presentó por medio de la activación del dispensador de agua. Cada cámara experimental se colocó dentro de un cubículo de aislamiento acústico (ENV-022M) con un ventilador que sirvió como ruido blanco y facilitó la circulación del aire al interior de la cámara. La programación, registro y colección de eventos se realizó mediante un equipo de cómputo, una interfase y el software MED-PC IV para ambiente Windows.

\section{Procedimiento}

Se usó un procedimiento de discriminación condicional como el reportado por Williams (1998), al inicio de cada ensayo se presentó una señal condicional (tono fijo o tono intermitente) que tuvo una duración de $5 \mathrm{~s}$, a cuyo término se introdujeron las palancas en la cámara experimental. Se reforzó con una gota de agua la respuesta a la palanca izquierda si antes se había presentado el tono fijo o la respuesta a la palanca derecha si antes se había presentado el tono intermitente (respuestas correctas); la respuesta a la palanca derecha después del tono fijo o la respuesta a la palanca de izquierda después del tono intermitente se consideraron como respuestas incorrectas. La ocurrencia de respuesta correcta o respuesta incorrecta retiraba inmediatamente ambas palancas hasta el siguiente ensayo. Cada sesión estuvo conformada por 100 ensayos (50 con tono fijo y 50 con tono intermitente). Los sujetos estuvieron en estas condiciones durante 24 sesiones.

En un segunda fase se mantuvieron las mismas condiciones que en la fase anterior (adquisición) excepto que la inserción de las palancas en la cámara experimental ocurrió $2 \mathrm{~s}$ después del término de la señal condicional (demora $2 \mathrm{~s}$ ). En fases sucesivas se incrementó la duración de la demora a 4 y $8 \mathrm{~s}$, cada fase estuvo vigente durante 5 sesiones. Después de la respuesta incorrecta o de la entrega de la gota de agua iniciaba el IEE. Para cuatro ratas el IEE se mantuvo en $45 \mathrm{~s}$ durante todo el experimento (IEE constante); mientras que para otras cuatro ratas se incrementó en 45, 90 y $180 \mathrm{~s}$ (IEE variable).

\section{RESULTADOS Y DISCUSIÓN}

En la Figura 1 se presenta el porcentaje de respuestas correctas por grupo en cada valor de la demora entre la señal condicional y la oportunidad para responder. En esta figura se puede observar que el grupo con el IEE constante se encontró un decremento abrupto en el porcentaje de respuestas 
correctas hasta el valor de demora de 8s; mientras que en el grupo con un IEE variable se observan decrementos mas graduales en el porcentaje de respuestas correctas a medida que se alargó la duración del intervalo de demora entre la señal condicional y la oportunidad para responder. Los sujetos de ambos grupos concluyeron la fase de adquisición (demora cero) con porcentajes de respuestas correctas superiores al $85 \%$, el incremento en la demora entre la señal condicional y la oportunidad de responder resultó en un claro efecto de tiempo relativo al observar un menor decremento en el porcentaje de respuestas correctas en los sujetos en los que se incrementó la duración del intervalo entre ensayos (Grupo Variable). Estos resultados son consistentes con los reportados por Williams (1998) y amplían su generalidad a situaciones en las que se evalúan los efectos de la demora entre la señal condicional y la oportunidad de respuesta (intervalo de retención) sobre condiciones de "estado estable", a diferencia de situaciones en las que se evalúan los efectos de la demora sobre la adquisición de discriminaciones condicionales demoradas.

Adicionalmente, los resultados son consistentes con los reportados en otros trabajos al mostrar que incrementos en el intervalo de demora entre la señal condicional y la oportunidad para responder resultan en decrementos en los porcentajes de respuestas correctas (Sargisson \& White, 2003; White, 1985, 2001; White \& Wixted, 1999, 2001; Wixted, 1989).

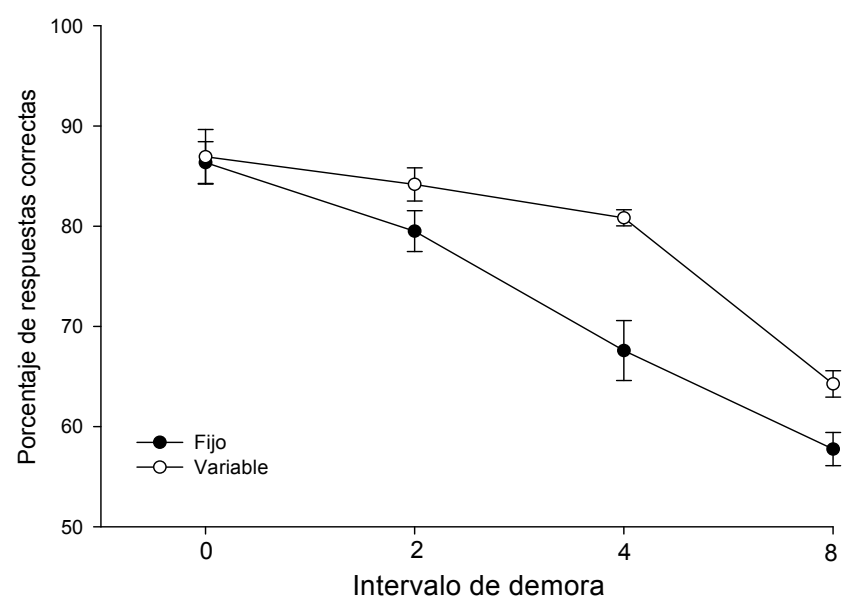

Figura 1. Porcentaje promedio de respuestas correctas de cada grupo en cada valor del intervalo de demora entre la señal condicional y la oportunidad para responder, las líneas verticales en cada uno de los puntos representan el error estándar. 
Sargisson y White (2003) entrenaron palomas en una tarea de igualación en la que se presentaban intervalos de demora que variaban desde 0 hasta 8 s y reportaron una menor precisión del responder (i.e., porcentaje de respuestas correctas) ante los valores mas grandes del intervalo de demora.

Williams (1998, Exp. 2 y 3) reportó que los efectos de la demora de reforzamiento sobre la adquisición de discriminaciones condicionales no se modificaba por el uso de diferentes duraciones del intervalo entre ensayos, es decir, que no se observó el ETR. En un intento por evaluar el ETR bajo condiciones de estado estable y no sobre condiciones de adquisición como en el estudio de Williams, es que en el Experimento 2 se replicó el mismo procedimiento que en el Experimento 1 excepto que se evaluaron los efectos de incrementar la demora de reforzamiento en lugar del intervalo de demora entre la señal condicional y la oportunidad de responder.

\section{EXPERIMENTO 2}

\section{MÉTODO}

Sujetos

8 ratas ingenuas experimentalmente y con las mismas características a las usadas en el Experimento 1.

Aparatos

Se utilizaron los mismos aparatos a los usados en el Experimento 1.

Procedimiento

El procedimiento fue el mismo al usado en el Experimento 1, excepto que se incrementó la demora de reforzamiento en 0, 2, 4 y $8 \mathrm{~s}$.

\section{RESULTADOS Y DISCUSIÓN}

En la Figura 2 se presenta el porcentaje de respuestas correctas para cada grupo en cada valor de la demora de reforzamiento. En esta figura se puede observar que los porcentajes de respuestas correctas se mantienen por arriba del $85 \%$ y prácticamente sin cambios ante los diferentes valores de la demora de reforzamiento. También se puede observar que en el grupo con IEE constante los porcentajes de respuestas correctas se encuentran por abajo de los observados en el grupo con IEE variable.

A diferencia de los resultados del Experimento 1 en el que se observaron decrementos en el porcentaje de respuestas correctas como resultado de 


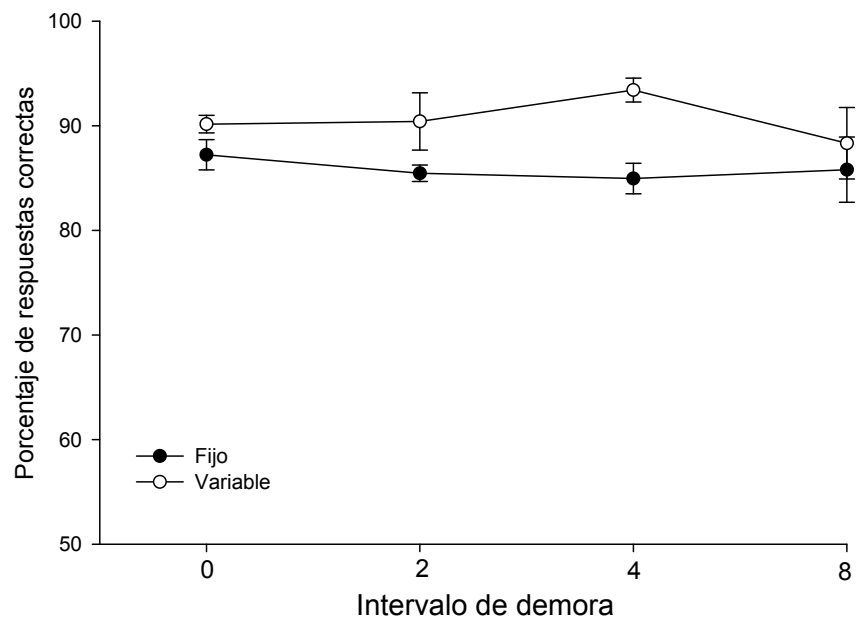

Figura 2. Porcentaje promedio de respuestas correctas de cada grupo en cada valor de la demora de reforzamiento, las líneas verticales en cada uno de los puntos representan el error estándar.

incrementar el intervalo de demora entre la señal condicional y la oportunidad para responder, en el Experimento 2 no se observaron los mismos efectos por el incremento en la demora de reforzamiento.

Los resultados del Experimento 2 son consistentes con los reportados por Williams (1998, Exp. 2 y 3) al no observar el ETR como resultado de alargar la demora de reforzamiento. No haber encontrado un decremento en el porcentaje de respuestas correctas en ninguno de los grupos es inconsistente con lo reportado en varios estudios en los que se ha documentado claramente un decremento en la precisión del responder como resultado de incrementar la demora de reforzamiento (e.g., D’Amato \& Cox, 1976; McCarthy \& Davison, 1986, 1991; Weavers, Foster \& Temple, 1998; Wilkie \& Spetch, 1978).

Por ejemplo, Wilkie y Spetch (1978) empleando palomas como sujetos evaluaron el efecto de la demora de reforzamiento sobre la precisión del responder en una tarea de igualación demorada. En una primera condición incrementaron el requisito de respuesta durante el estímulo de comparación (desde 1 hasta 16 respuestas) como una manera de incrementar la demora de reforzamiento; posteriormente sustituyeron el requisito de respuesta por demoras nominales de reforzamiento que se incrementaron desde 0 hasta 9 s. Ambas manipulaciones, tanto incrementar el requisito de respuesta como 
la duración de la demora nominal de reforzamiento resultaron en decrementos en la precisión del responder, es decir, decrementos en el porcentaje de respuestas correctas. Estos resultados son consistentes a los reportados por D'Amato y Cox (1976) al evaluar los efectos de la demora de reforzamiento utilizando una tarea de igualación a la muestra y monos como sujetos.

\section{DISCUSIÓN GENERAL}

El propósito del trabajo fue evaluar el efecto conjunto de variar la duración del intervalo entre ensayos y el intervalo de demora entre la señal condicional y la oportunidad para responder (Experimento 1) y de la demora de reforzamiento (Experimento 2) sobre el porcentaje de respuestas correctas en una tarea de discriminación condicional.

Como se señaló anteriormente, el ETR se ha reportado empleando diferentes procedimientos y situaciones experimentales. En situaciones de automoldeamiento (i.e. que implican contingencias estímulo-estímulo) se sabe que la velocidad de adquisición o establecimiento de la respuesta automoldeada depende de la duración del ciclo $(C)$ relativa a la duración del ensayo (T), es decir, de la razón C/T (e.g., Balsam, 1984; Bueno \& Álvarez, 2001). En el campo de la elección y utilizando programas concurrentes encadenados, también se ha reportado que el responder de los organismos está determinado o regulado por la duración del componente inicial relativo a la duración del intervalo promedio entre reforzadores (e.g., Fantino, 1977; Squires \& Fantino, 1971).

Los resultados del Experimento 1 son consistentes con los reportados por Williams (1998) al observar que los efectos de incrementar la duración del intervalo de demora se vieron modificados por la duración del intervalo entre ensayos. También son consistentes con los resultados de otros trabajos sobre adquisición de discriminaciones condicionales (e.g., Holt \& Shafer, 1973; Roberts \& Kreamer, 1982). Los presentes resultados extienden la generalidad del efecto de tiempo relativo a situaciones que implican el mantenimiento de la conducta y no únicamente a situaciones de adquisición.

Los resultados del Experimento 2 no son consistentes con los observados en el Experimento 1 y no fácilmente pueden ser integrados a la literatura de tiempo relativo dado que no se encontraron decrementos en el porcentaje de respuestas correctas por incrementar la demora de reforzamiento. La ausencia de este efecto restringe la posibilidad de extender el ETR a situaciones en las que se alteran las relaciones de contingencia respuesta-reforzador.

Es posible que el tipo de tarea, en este caso el uso de un procedimiento de discriminación condicional, imprima diferencias importantes respecto de 
otros procedimientos en los que variando la demora de reforzamiento se ha reportado el ETR (e. g., Schaal, Schuh \& Branch, 1992).

Resulta intrigante observar que no hay cambios sistemáticos en el responder como resultado de incrementar la demora de reforzamiento. En los estudios en los que se han evaluado los efectos de la demora de reforzamiento se han reportado decrementos en la frecuencia o tasa de respuesta asociados a incrementos en la demora de reforzamiento, lo que en la literatura se ha reportado como gradiente de demora (Flores \& Mateos, 2009; Lattal, 1987; Perin, 1943; Pulido, Paz \& Sosa, 2008; Pulido, Sosa \& Valadez, 2006; Renner, 1964; Ruiz \& Bruner, 2008; Tarpy \& Sawabini, 1974). A diferencia de los estudios referidos, en el presente estudio se registró la precisión del responder (i.e porcentaje de respuesta correctas), es posible que la demora de reforzamiento tenga un fuerte impacto sobre la persistencia del responder (i. e. tasa o frecuencia de respuesta) pero no sobre su precisión (i. e. índice de discriminación o porcentaje de respuestas correctas).

Los anterior puede verse apoyado o fortalecido dado que en algunos estudios se ha reportado que empleando procedimientos de igualación de la muestra, el incremento en la demora de reforzamiento tiene un moderado efecto sobre la precisión del responder, mientras que la demora entre la señal condicional y la oportunidad para responder resulta en un decremento progresivo en el porcentaje de respuesta correctas (e.g., Sargisson \& White, 2003).

Por ejemplo, Sargisson y White (2003) evaluaron los efectos de variar la demora de reforzamiento y la demora entre la señal condicional y la oportunidad para la respuesta (intervalo de retención). Variaron entre sesiones la duración del intervalo de retención con valores que oscilaron desde 0 hasta 8 $\mathrm{s}(0,2,4,6$ y $8 \mathrm{~s})$; mientras que entre fases variaron la duración de la demora de reforzamiento usando los mismos valores que en el caso del intervalo de retención $(0,2,4,6$ y 8 s). Reportaron decrementos en la precisión del responder tanto por alargar la duración del intervalo de retención como por incrementar la demora de reforzamiento; sin embargo, los incrementos en el intervalo de retención resultaron en decrementos más marcados en la precisión, que los decrementos en el porcentaje de respuestas correctas producidos por alargar la demora de reforzamiento.

En otro trabajo, McCarthy y Davison (1986) utilizando una tarea de igualación a la muestra reportaron los efectos de variar la demora entre el estímulo muestra y la oportunidad para responder a los estímulos de comparación (intervalo de retención) y la demora de reforzamiento, de manera consistente con los resultados reportado por Sargisson y White (2003), encontraron que ambas manipulaciones resultaron en un decremento en la precisión del responder y que nuevamente el decremento en la precisión al incrementar la demora de reforzamiento fue menor que el decremento en la precisión como resultado de alargar la duración del intervalo de retención. 
En su conjunto, los resultados del presente estudio extienden la generalidad del ETR a situaciones diferentes de adquisición, específicamente en el caso de la variación en la demora entre la señal condicional y la oportunidad para responder (Exp. 1). Sin embargo los resultados de variar la demora de reforzamiento (Exp.2) son difíciles de integrarse como un caso de ETR. Al respecto Williams $(1998,2003)$ ha sugerido que los efectos de variar la demora de reforzamiento y la demora entre la señal condicional y la oportunidad para responder son el resultado de procesos cualitativamente diferentes.

La generalidad de los resultados reportados en la literatura, con excepción de algunos trabajos (e. g., Schaal, Schuh \& Branch ,1992), muestran que tanto en situaciones de adquisición como en condiciones de mantenimiento, los efectos que resultan de incrementar la demora de reforzamiento no son consistentes con el principio de tiempo relativo (e. g., Bruner, Avila, Acuña \& Gallardo, 1998; Shahan \& Lattal, 2005).

Ante la inconsistencia de resultados por variar la demora de reforzamiento, recientemente Ruiz, Bruner y Balderrama (2007) reportaron un trabajo en el que evaluaron los efectos de la demora de reforzamiento variando la probabilidad de que el intervalo respuesta-reforzador estuviera señalado o no señalado. Un grupo de sujetos se mantuvo con un intervalo entre reforzadores de $32 \mathrm{~s}$, mientras que en otro grupo el intervalo entre reforzadores fue de $128 \mathrm{~s}$. Variaron entre condiciones el valor de probabilidad de la señal $(0.0$, $0.33,0.66$ y 1.0$)$, mientras que los valores del intervalo de demora de reforzamiento $(0,2,4$ y 8 s) se variaron intra sesión. Ruiz et al. (2007) reportaron el ETR en el grupo con el intervalo entre reforzadores de $128 \mathrm{~s}$, especialmente cuando el intervalo de demora de reforzamiento estuvo señalado con una alta probabilidad (1.0).

Aún cuando el estudio de Ruiz et al. (2007) representa una manipulación sistemática que permitió evaluar los efectos de la demora de reforzamiento en la que se transita de una situación con demora no señalada a una con demora señalada, y con ello contribuir a identificar las condiciones para observar el ETR, sigue siendo intrigante por qué bajo las condiciones del presente estudio, en particular las del Experimento 2 no se observaron cambios en el responder como resultado de incrementar la demora de reforzamiento.

Las explicaciones ofrecidas ante la diferencia de resultados por variar la demora de reforzamiento y la demora entre la señal condicional y la oportunidad para responder han atendiendo a una pérdida de discriminabilidad. En este sentido, Davison y Nevin (1999) ofrecieron un modelo para explicar las ejecuciones en tareas de discriminación condicional, asumiendo que la precisión del responder está determinada tanto por la discriminabilidad entre los estímulos (intervalo de retención) como por la discriminabilidad entre las contingencias de reforzamiento (demora de reforzamiento), haciendo predicciones de diferentes ejecuciones que resultan de la interacción entre los 
efectos de la demora de reforzamiento y del intervalo de retención. En este modelo, la reducción en la discriminabilidad por incrementar la demora de reforzamiento es atribuida a un decremento o degradación de la contingencia respuesta-reforzador.

La ausencia de decrementos en el porcentaje de respuestas correctas por incrementar la demora de reforzamiento (Experimento 2) sigue siendo un resultado difícil de interpretar a la luz del modelo propuesto por Davison y Nevin. Es posible que el decremento en el porcentaje de respuestas correctas (Experimento 1) y que el mantenimiento en la precisión ante los diferentes valores de demora de reforzamiento (Experimento 2) pueda deberse a que los intervalos temporales estímulo-respuesta y respuesta-reforzador operen de manera diferente. Por ejemplo, Davison y Jenkins (1985) señalaron que cuando se incrementa la demora entre el estímulo y la oportunidad para responder se pierde gradualmente el control discriminativo que tiene la señal al momento ante el cual el sujeto tiene que emitir la respuesta de elección entre las alternativas; mientras que cuando se incrementa la demora de reforzamiento, el control por el reforzador subsecuente a la respuesta de elección se reduce, de este modo los incrementos en el intervalo respuesta-reforzador son entendidos como una degradación del control que tiene el reforzador de la respuesta de la que es contingente el evento de estímulo.

Como se mencionó en la sección introductoria, existen discrepancias en los efectos de incrementar la demora de reforzamiento en situaciones en las que se tienen diferentes frecuencias de reforzamiento. Por ejemplo, Shahan y Lattal (2005) no encontraron efectos diferenciales por introducir una demora de reforzamiento al variar la frecuencia de reforzamiento mediante los valores de dos programas de intervalo variable (IV20 s -IV120 s), intentando evaluar con ello si los efectos de la demora entre la respuesta y el reforzador se regulaban por el intervalo promedio entre reforzadores (i.e., efecto de tiempo relativo). Los resultados reportados por Shahan y Lattal, así como los reportados por Bruner, Ávila, Acuña y Gallardo (1998) y los del presente estudio (Experimento 2) son consistentes y apoyan las conclusiones de Williams (1998) de que el efecto de tiempo relativo no ocurre cuando se introduce un intervalo entre la respuesta y el reforzador.

La interpretación ofrecida por Wiilliams $(1998,2003)$ al identificar un efecto de tiempo relativo por variar el intervalo de retención y a su ausencia por introducir una demora de reforzamiento se restringió a mencionar que los efectos de ambas variables tienen propiedades funcionales distintas y que pueden reflejar diferencias fundamentales en los procesos psicológicos implicados.

Futuras exploraciones sistemáticas son necesarias para contribuir a identificar la discrepancia e inconsistencia entre los resultados por incrementar el intervalo de demora estímulo-respuesta (intervalo de retención) y respuesta- 
reforzador (demora de reforzamiento) en situaciones en las que existen diferentes frecuencias de reforzamiento, buscando con ello elaborar una explicación satisfactoria de la ausencia del efecto de tiempo relativo cuando se degrada la contingencia respuesta-reforzador.

\section{REFERENCIAS}

Balsam, P. (1984). Relative time in trace conditioning. Annals of the New York Academy of Sciences, 423, 211-227.

Bruner, C., Avila, R., Acuña, L. \& Gallardo, L. (1998). Effects of reinforcement rate and delay on the acquisition of lever pressing by rats. Journal of the Experimental Analysis of Behavior, 69, 59-75.

Bueno, M. \& Álvarez, R. (2001). El efecto de las duraciones de intervalos entre ensayos y entre estímulos en el condicionamiento pavloviano apetitivo en ratas. Psicológica, 22, 205-215.

D’Amato, M.R. \& Cox,J.K. (1976). Delay of consequences and short-term memory in monkeys. En D.L. Medin, W.A. Roberts, \& R.T. Davis (Eds.), Processes of animal memory (pp.49-78). Mahwah, NJ:Erlbaum.

Davison, M. \& Jenkins, P.E. (1985). Stimulus discriminability, contingency discriminability, and schedule performance. Animal Learning and Behavior, 13, 77-84.

Davison, M. \& Nevin, J. (1999). Stimuli, reinforcers, and behavior: An integration. Journal of the Experimental Analysis of Behavior, 71, 439-482.

Fantino, E. (1977). Conditioned reinforcement: Choice and information. En W.K. Honig \& J.E.R. Staddon (Eds.), Handbook of operant behavior (pp.313-339). Englewood Cliffs, NJ:Prentice Hall.

Flores, C. \& Mateos, R. (2009). Adquisición del responder bajo condiciones de reforzamiento demorado en programas temporales señalados. Universitas Psychologica, 8, 249-258.

Gibbon, J., Baldock, M.D., Locurto, C., Gold, L., \& Terrace, H.S. (1977). Trial and intertribal durations in autoshaping. Journal of Experimental Psychology: Animal Behavior Processes, 3, 264-284.

Gibbon, J., \& Balsam, P. (1981). Spreading association in time. En C.M. Locurto, H.S. Terrace, \& J. Gibbon (Eds.), Autoshaping and conditioning theory (pp. 219-253). New York: Academic Press.

Holt, G.L. \& Shafer, J.N. (1973). Function of intertrial interval in matching to sample. Journal of the Experimental Analysis of Behavior, 19, 181-186.

Lattal, K.A. (1987). Considerations in the experimental analysis of reinforcement delay. En M.L. Commons, J.E. Mazur, J.A. Nevin \& H. Rachlin (Eds.) Quantitative analysis of behavior: Vol. 5. The effect of delay and intervening events on reinforcement value (pp. 107-123). Hillsdale, NJ: Erlbaum.

Maki, W.S., Moe, J.C., \& Bierly, C.M. (1977). Short-term memory for stimuli, responses, and reinforcers. Journal of Experimental Psychology: Animal Behavior Processes, 3, 156-177. 
McCarthy, D. \& Davison, M. (1986). Delayed reinforcement and delayed choice in symbolic matching to sample: Effects on stimulus discriminability. Journal of the Experimental Analysis of Behavior, 46, 293-303.

Perin, C.T. (1943). A quantitative investigation of the delay-of-reinforcement gradient. Journal of Experimental Psychology, 32, 37-51.

Pulido, M., Paz, M., \& Sosa, R. (2008). The effects of behavioral history on response acquisition with delayed reinforcement: A parametric analysis. Revista Mexicana de Análisis de la Conducta, 34, 43-56.

Pulido, M., Sosa, R., \& Valadez, L. (2006). Adquisición de la operante libre bajo condiciones de reforzamiento demorado: Una revisión. Acta Comportamentalia, 14, 5-21.

Renner, K.E. (1964). Delay of reinforcement: A historical review. Psychological Bulletin, 61, 341-361.

Roberts, W.A., \& Kraemer, P.J. (1982). Some observations of the effect of intertribal interval and delay on delayed matching to sample in pigeons. Journal of Experimental Psychology: Animal Behavior Processes, 8, 342-353.

Ruiz, J.A., \& Bruner, C. (2008). Demora de reforzamiento con agua en un procedimiento de beber inducido por el programa. Revista Mexicana de Análisis de la Conducta, 34, 97-109.

Ruiz, J.A., Bruner, C. \& Balderrama, D.M. (2007). Efecto de tiempo relativo en demoras de reforzamiento señaladas y no señaladas. Revista Mexicana de Análisis de la Conducta, 33, 119-138

Sargisson, R.J. \& White, K.G. (2003). The effect of reinforcer delays on the form of the forgetting function. Journal of the Experimental Analysis of Behavior, 80, 77-94.

Schaal, D.W., Schuh, K.J., \& Branch, M.N. (1992). Key pecking of pigeons under variable interval schedules of briefly signaled delayed reinforcement: Effects of variable-interval value. Journal of the Experimental Analysis of Behavior, 58, 277286.

Shahan, T. \& Lattal, K.A. (2005). Unsignaled delay of reinforcement, relative time, and resistance to change. Journal of the Experimental Analysis of Behavior, 83, 201-219.

Squires, N., \& Fantino, E. (1971). A model for choice in simple concurrent and concurrent-chains schedules. Journal of the Experimental Analysis of Behavior, 15, 27-38.

Tarpy, R.M. \& Sawabini, F.L. (1974). Reinforcement delay: A selective review of the last decade. Psychological Bulletin, 81, 984-997.

Weavers, R., Foster, T.M., \& Temple, W. (1998). Reinforcer efficacy in a delayed matching-to-sample task. Journal of the Experimental Analysis of Behavior, 69, 7785.

White, K.G. (1985). Characteristics of forgetting functions in delayed matching to sample. Journal of the Experimental Analysis of Behavior, 44, 15-34.

White, K.G. (2001). Psychophysics of direct remembering. En. M.L. Commons, J.A. Nevin \& M.C. Davison (Eds.), Signal detection: Mechanisms, models and applications (pp.221-237). Mahwah, NJ:Erlbaum.

White, K.G., \& Wixted, J. T. (1999). Psychophysics of remembering. Journal of the Experimental Analysis of Behavior, 71, 91-113. 
White, K.G., \& Wixted, J. T. (2001). Psychophysics of remembering. The General Psychologist, 36, 56-63.

Wilkie, D.M., \& Spetch, M.L. (1978). The effect of sample and comparison ratio sachedules on delayed matching to sample in the pigeon. Animal Learning \& Behavior, 6, 273-278.

Williams, B. A. (1998) Relative time and delay of reinforcement. Learning and motivation. 29, 236-248.

Williams, B.A. (2003). Challenges to timing-based theories of operant behavior. Behavioural Processes, 62, 115-123.

Wixted, J.T. (1989). Nonhuman short-memory: A quantitative reanalysis of selected findings. Journal of the Experimental Analysis of Behavior, 52, 409-426.

Yin, H., Barnet, R.C., \& Miller, R.R. (1994). Trial spacing and trial distribution effect in Pavlovian conditioning: Contributions of a comparator mechanism. Journal of Experimental Psychology, 20, 123-134. 